\title{
Multi-Output Compliant Shape Memory Alloy Bias-Spring Actuators
}

\author{
Sean Thomas*, Adrien Thabuis*, Thomas Martinez and Yves Perriard ${ }^{1}$, Senior Member, IEEE
}

\begin{abstract}
Smart Materials such as Shape Memory Alloys (SMA) play a major role in creating Smart Actuators that are compact and high performing. Traditional SMA bias-spring actuators are only capable of a single linear output. Using topology optimization, compliant SMA bias-spring mechanisms that can create multi-output displacements from a single biasspring are designed to improve upon the traditional actuators. These new smart actuators are then simulated using Finite Element Modelling to validate the presence of the Shape Memory Effect.
\end{abstract}

Index Terms-smart material, topology optimization, computer-aided design, finite element analysis

\section{INTRODUCTION}

Technology has been steadily pushing towards smaller and more compact devices in recent decades. This has created a vacuum for materials that can provide the most work with the least amount of material. Smart materials, in general, have begun to play an essential role in filling that void. Shape Memory Alloys (SMA), especially, are an ideal candidate due to their high volumetric work density [1].

SMAs are a type of Smart Material that react to heat. Their interesting behaviour of recovering any initial strain placed upon then when heated has been exploited to created compact and powerful actuators. Their remarkable strain recovery behavior, while promising, is quite difficult to model and control. This makes it especially difficult to design actuators that are complex and multi-dimensional. Generally, most SMA actuators are created from simple geometries such as wires [2], [3], springs [4] and sheets [5].

Compliant mechanisms are another innovative area that has been crucial in designing complex structures that can perform complex output displacements without increasing the complexity of assembly or fabrication. On the other hand, designing such mechanisms is not a simple feat. It can either be done with the conventional design approach that relies on the designer's experience and mathematical models [6][8]; or either with various computational-aided methods that formulate the design problem as an optimization one. Among them, the so-called Topology Optimization Method (TOM) has been subject to a large research interest over the past years [9], [10]. It is a powerful and versatile design approach that directly investigates the distribution of material inside a discretized design space all the while improving an objective.

Combining the two fields to create a compliant mechanism fabricated from SMA has been explored in the work

\footnotetext{
*These authors contributed equally to this work

${ }^{1}$ These authors are with the Integrated Actuators Laboratory, Ecole Polytechnique Fédérale de Lausanne (EPFL), Switzerland yves.perriarddepfl.ch
}

shown in [11]. The work demonstrates that compliant SMA mechanisms do in-fact show the shape memory effect when deformed and heated. This allows for the direct integration of a bias-spring into the compliant SMA mechanism to create a compact actuator with a complex output. This brings about a new area of SMA actuator design that can improve upon the standard bias-spring SMA actuator.

This work, with the help of topology optimization, shows the potential of these compliant SMA bias-spring mechanisms that can produce multi-output displacements using only a single bias-spring. The designs presented in this work have also been simulated using commercially available Finite Element Modelling (FEM) software, ANSYS, to demonstrate the presence of the Shape Memory Effect (SME) when heated.

\section{Shape Memory EFFect}

\section{A. Shape Memory Alloys}

Shape Memory Alloys are a class of smart materials that respond to heat. They have the very interesting ability to recover any strain it was subjected to when exposed to temperatures higher than a certain threshold. This fascinating behaviour occurs due to phase transformations and the fact that the material exists in distinct phases with respect to temperature.

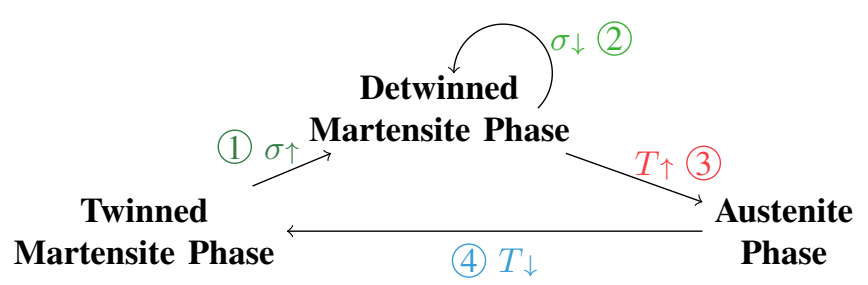

Fig. 1: Phase transformations of Shape Memory Alloys depending on the applied stress $\sigma$ and temperature $T$.

In Fig. 1, the different phases of the material are presented. The material exists in three distinct phases based on stress and temperature. At low temperatures, the material exists in the Martensite (M) phase. When a certain level of stress (critical detwinning stress) is applied to the material, it changes from the Twinned $\mathrm{M}$ phase to the Detwinned $\mathrm{M}$ phase (1). In this phase, the material keeps its new shape even when the previously applied stress is released (2). This can be referred to as activating the material. Only when the material is detwinned, will it revert back to its original shape (3) when heated. Finally, when the material is cooled down 
(4), the material revert back to the twinned M phase where the cycle can be renewed.

\section{B. Biased-spring actuators}

Due to the high energy density of SMAs [1], they are a prime candidate for compact and high work output actuators. Since SMAs require a stress to be applied for the detwinning process, some kind of biasing element must be integrated to create an SMA actuator. For example, a dead weight, spring or another SMA element can be used to deform the SMA at low temperatures to activate the material and then when heat is applied, the SMA will lift the dead weight, or deform the attached spring/antagonistic SMA element [12].

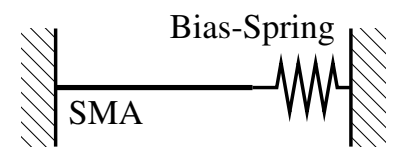

Fig. 2: A schematic for a Bias-Spring SMA actuator.

Since the behaviour of the springs is well studied, they are an ideal candidate to be paired with SMAs to create an actuator. Thus, bias-spring SMA actuators have become a common type of SMA actuators [12]. Here, the SMA is attached to a bias-spring that will deform the smart material at low temperatures. Conversely, the spring is deformed by the SMA at high temperatures to create a reversible linear actuation as shown in Fig. 2. But because the behaviour of SMAs is quite complicated and difficult to model, the SMA elements in the actuator are usually made in simple geometries such as wires, springs or sheets. Designing the material in more complex structures will make it difficult to model and predict the resulting shape memory behaviour. In cases where complex output displacements are required, transducers are attached to transform the linear output of the SMA Bias-Spring actuator [13]. Most work focuses on adapting multiple simple SMA wires and bias-springs with complex transducers to perform the desired complex outputs. This complicates the fabrication and assembly of the actuator rendering the actuator no longer a compact solution [14]. By designing an entire compliant bias-spring mechanism in the SMA material, a complex output can be achieved using a single bias-spring.

\section{Design Method}

The Topology Optimization Method (TOM) is used to design the biased-spring compliant mechanisms. It investigates the distribution of material inside a discretized design domain so as to improve an objective function. An analysis taking into account the SME of the material would incur a high computational cost. In the hopes to reduce this cost, an abstraction of its thermal properties is done. By optimizing purely for strain of the compliant mechanism design, the output direction can be indirectly taken into account as well as maximizing the distribution of strain overall in the mechanism. This will indirectly maximize the amount of material that can attain the detwinning which enables the shape memory effect.

The framework used in [11], is implemented and extended to Multi-Input Multi-Output (MIMO) problems with $N I$ inputs and $N O$ outputs as done in [15]. This results in multiobjective optimization with $N I \times N O$ sub-objectives for each input-output pair. They are defined as the ratio between the mutual strain energy $\mathrm{S}_{\text {mut }}^{i j}$ between the input $i$ and the output $j$ over the sum of the $i^{\text {th }}$ input strain energy: $\mathrm{S}_{\mathrm{in}}^{i}$ and the $j^{\text {th }}$ output one: $\mathrm{S}_{\text {out }}^{i}$. The minus sign comes from the minimization formulation of the optimization problem and that the maximization of the mutual strain energy is desired.

$$
\begin{gathered}
f=\sum_{i=1}^{\mathrm{NI}} \sum_{j=1}^{\mathrm{NO}} \omega_{i, j} \cdot f_{i j}, \\
f_{i j}=-\frac{\mathrm{S}_{\mathrm{mut}}^{i j}}{\mathrm{~S}_{\mathrm{in}}^{i}+\mathrm{S}_{\mathrm{out}}^{j}}=-\frac{\mathrm{U}_{\text {out }}^{j} \mathrm{KU}_{\mathrm{int}}^{i}}{\frac{1}{2} \mathrm{U}_{\text {out }}^{j} \mathrm{KU}_{\text {out }}^{j}+\frac{1}{2} \mathrm{U}_{\text {in }}^{i \mathrm{~T}} \mathrm{KU}_{\text {in }}^{i}} .
\end{gathered}
$$

$\mathrm{K}$ is the stiffness matrix of the current design and, as the distribution of material changes, is updated between iterations of the optimization. $\mathrm{U}_{\mathrm{in}}^{i}$ and $\mathrm{U}_{\text {out }}^{j}$ correspond to the nodal displacement of the design when the structure is subject to only $\mathrm{F}_{\text {in }}^{i}\left(i^{\text {th }}\right.$ input force $)$ and $\mathrm{F}_{\text {out }}^{j}\left(j^{\text {th }}\right.$ dummy output force $)$ respectively. $N I+N O$ Finite Element Analyses (FEA) are required to build the full objective function which solve $\mathrm{KU}_{\text {in }}^{i}=\mathrm{F}_{\text {in }}^{i}$ and $\mathrm{KU}_{\text {out }}^{j}=\mathrm{F}_{\text {out }}^{j}$ for $i=1, \ldots, N I$ and $j=$ $1, \ldots, N O$. The weights $\omega_{i, j}$ balance the trade-off between the different input-output pairs. To avoid manually tuning these weights, an auto-adapting scheme is implemented [16]. The ratios between the different sub-objectives are set to one in this research but different values could be desired so as to impose different magnitudes between the outputs displacements. More details about the formulation of the implemented topology optimization are presented in [11].

Topology optimization of compliant mechanisms often requires the use of artificial springs at the loaded ports: at the inputs to provide information on the input work imposed by the load, and at the output for the resistance provided by the work-piece that will be placed there. In the context of this research, the artificial springs at the input used during the design process and the biasing spring element used to form the actuator should not be confused together and are two different entities.

The symmetry axes can be incorporated inside the optimization as rolling support boundaries. This allows proceeding the optimization on a restricted design space instead of the whole domain. This can be better seen on Fig. 3, defining the design problem of a biased-spring 4-point mandrel, with the final topology resulting from the optimization. The design is restricted to a quarter of the design domain as it is symmetric two times. The evolution of the topology throughout the optimization is shown in Fig. 4.

The authors suggest to always place the biasing-spring (input force) on a rolling support or symmetry axis as it assures the spring stays along the same axis as it deforms. 

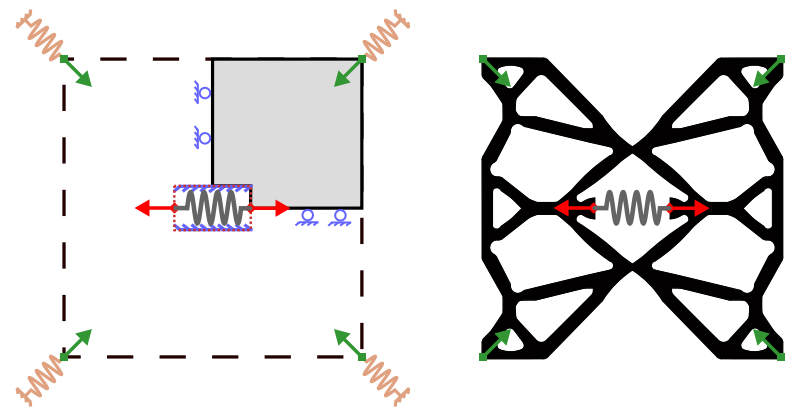

Fig. 3: 4-point mandrel: Design problem (left), Interpolated final topology (right). Input forces are shown in red while the output forces are shown green.

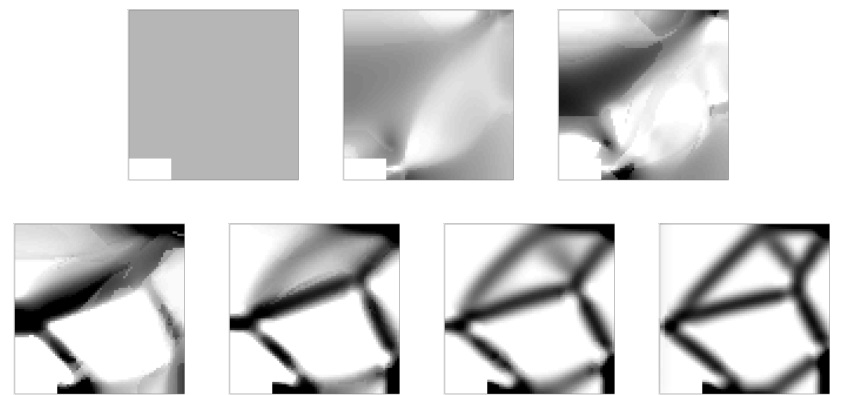

Fig. 4: 4-point mandrel: Evolution throughout the optimization. The grayscale represent the value of the filtered artificial density.
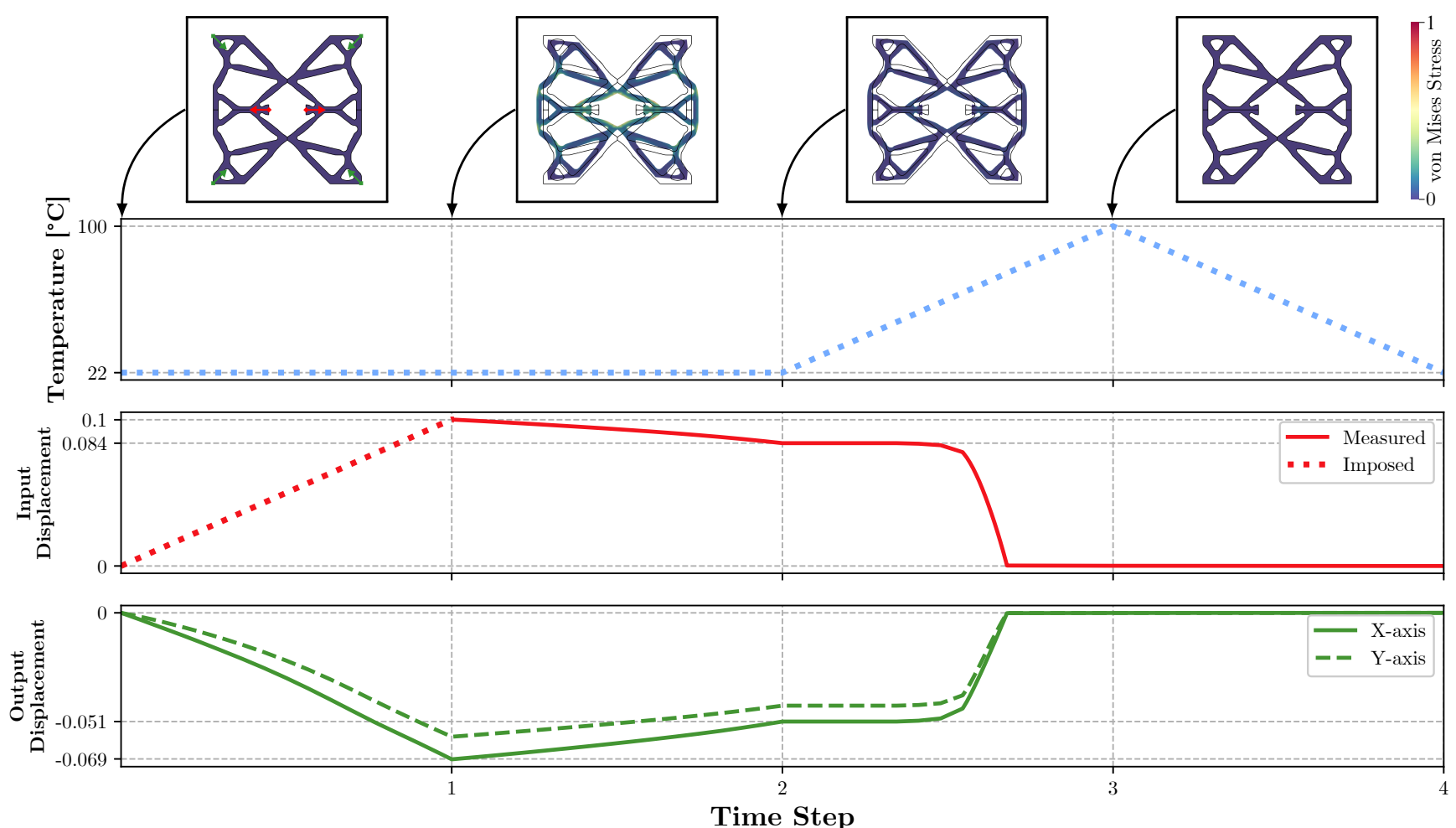

Fig. 5: Results of the shape memory effect simulation of the 4-point mandrel. Here, the displacements are calculated relatively to the length of a quarter of the design domain: $L / 2$. The displacements observed between time steps 1 and 2 show a strain retention of $\alpha_{\varepsilon}=84 \%$ confirming the presence of the shape memory effect.

\section{RESULTS}

In this section, various design problems with multiple outputs are studied. For the sake of simplicity, problems with a single input (single bias-spring with symmetric behavior) are treated, but the proposed approach can be applied to multiinput problems with multiple bias-springs. The examples shown are generated using a qualitative analysis where the exact dimensions are not taken into account as often done in topology optimization [9]. When fabricating a prototype, the dimensions of the designs can be scaled based on the force requirements of the application.

The implemented optimizer is the Method of Moving Asymptotes (MMA) [17] and a sensitivity filter is used to regulate the optimization problem and impose a minimal proximity length between two edges of the structure [9]. A volume infill limitation of the design domain constrains the optimization problem and is set to $30 \%$. The analysis of the shape memory effect consist of four time steps and is shown for the different actuators in Figs. 5, 6 and 8. It proceeds as follows: an input displacement is first applied, generating a displacement at the output. If every deformed area has undergone the detwinning process, the structure should keep the deformed shape when the input displacement is released after the first time step. However, as some parts of the compliant mechanism are not strained enough, the level of detwinning within the structure is not uniform and a 

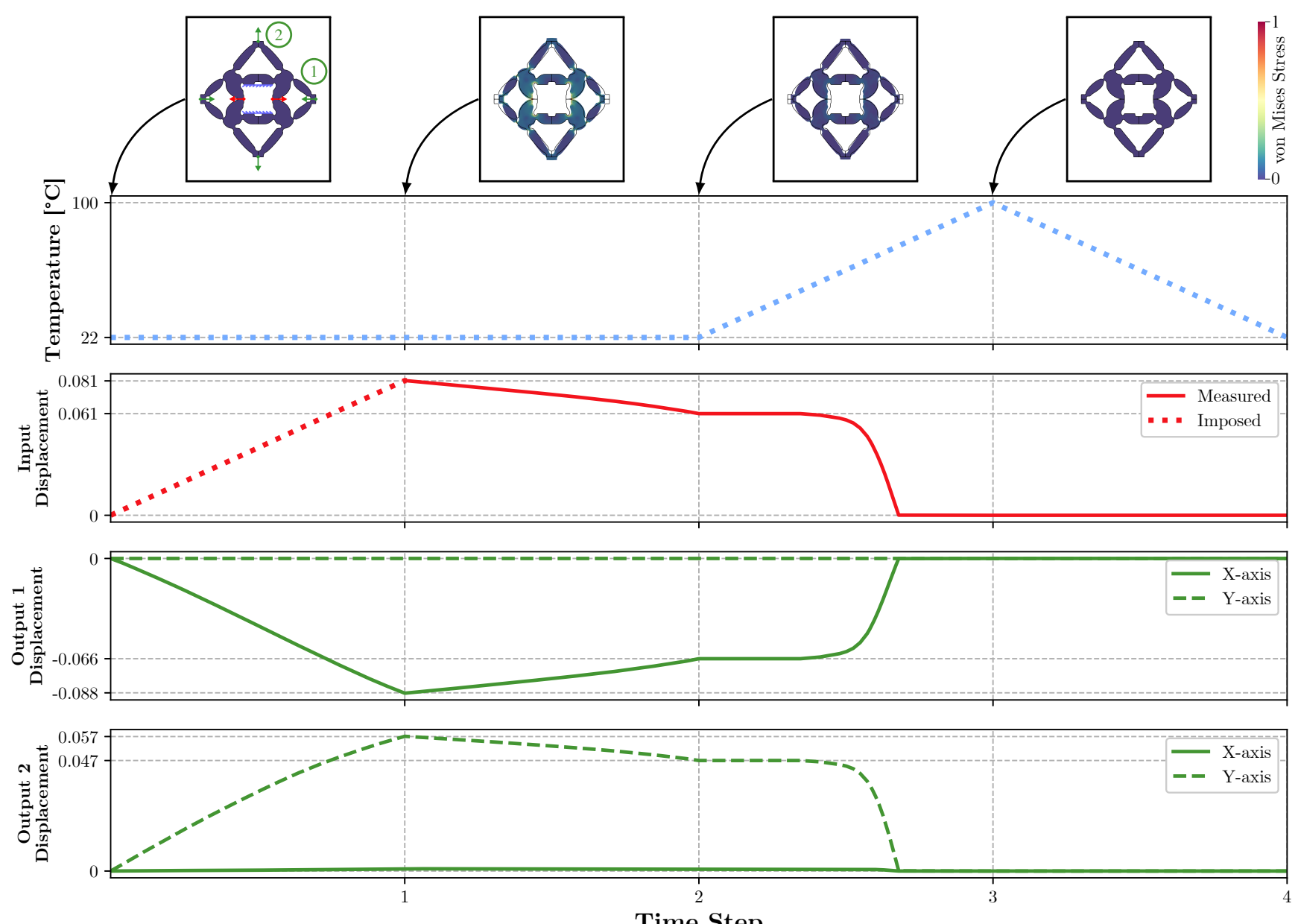

Time Step

Fig. 6: Results of the shape memory effect simulation of the rhombus actuator. Here, the displacements are calculated relatively to the length of a quarter of the design domain: $L / 2$. The displacements observed between time steps 1 and 2 show a strain retention of $\alpha_{\varepsilon}=75.6 \%$ confirming the presence of the shape memory effect. The output displacements are numbered based on Fig. 7.

fraction of the strain will be lost when the input displacement is released. During the third time step, the structure is heated to revert it back to its original shape. Finally, the structure is cooled down to return the material to its initial phase.

The strain retention $\alpha_{\varepsilon}$ seen at the end of the second time step, as proposed in the work [11], can be used as a quantitative factor to evaluate the ability of the design to be fabricated as an actuator. It is defined as :

$$
\alpha_{\varepsilon}=1-\frac{\varepsilon_{\text {loaded }}-\varepsilon_{\text {free }}}{\varepsilon_{\text {loaded }}},
$$

with $\varepsilon_{\text {loaded }}$ and $\varepsilon_{\text {free }}$ representing the domain space normalised input displacement at time step 1 and 2 respectively (as seen in Figs. 5, 6 and 8). This factor varies between $\alpha_{\varepsilon}=0$ implying that no SME has been observed, and $\alpha_{\varepsilon}=1$ implying that all the parts of the structure contributing to the multi-output displacement have detwinned.

The first example consists of a mandrel-like structure in which the bias-spring activates the four corners of the domain space (of size $L \times L$ ) to contract radially towards the center as seen in Fig. 3. The results of the ANSYS simulation showing
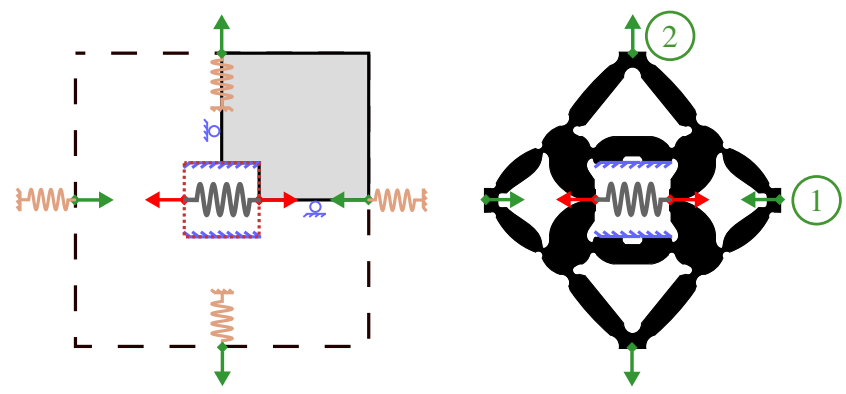

Fig. 7: Rhombus mechanism: Design problem (left), Interpolated final topology (right). Input forces are shown in red while the output forces are shown green.

the SME can be observed in Fig. 5. The second example consists of a Rhombus structure contained into a squared design domain, again, of size $L \times L$, as seen in Fig. 7 . Here, the bias-spring activates a displacement of 4 outputs that elongate in the $y$-axis and contract in the x-axis. The 

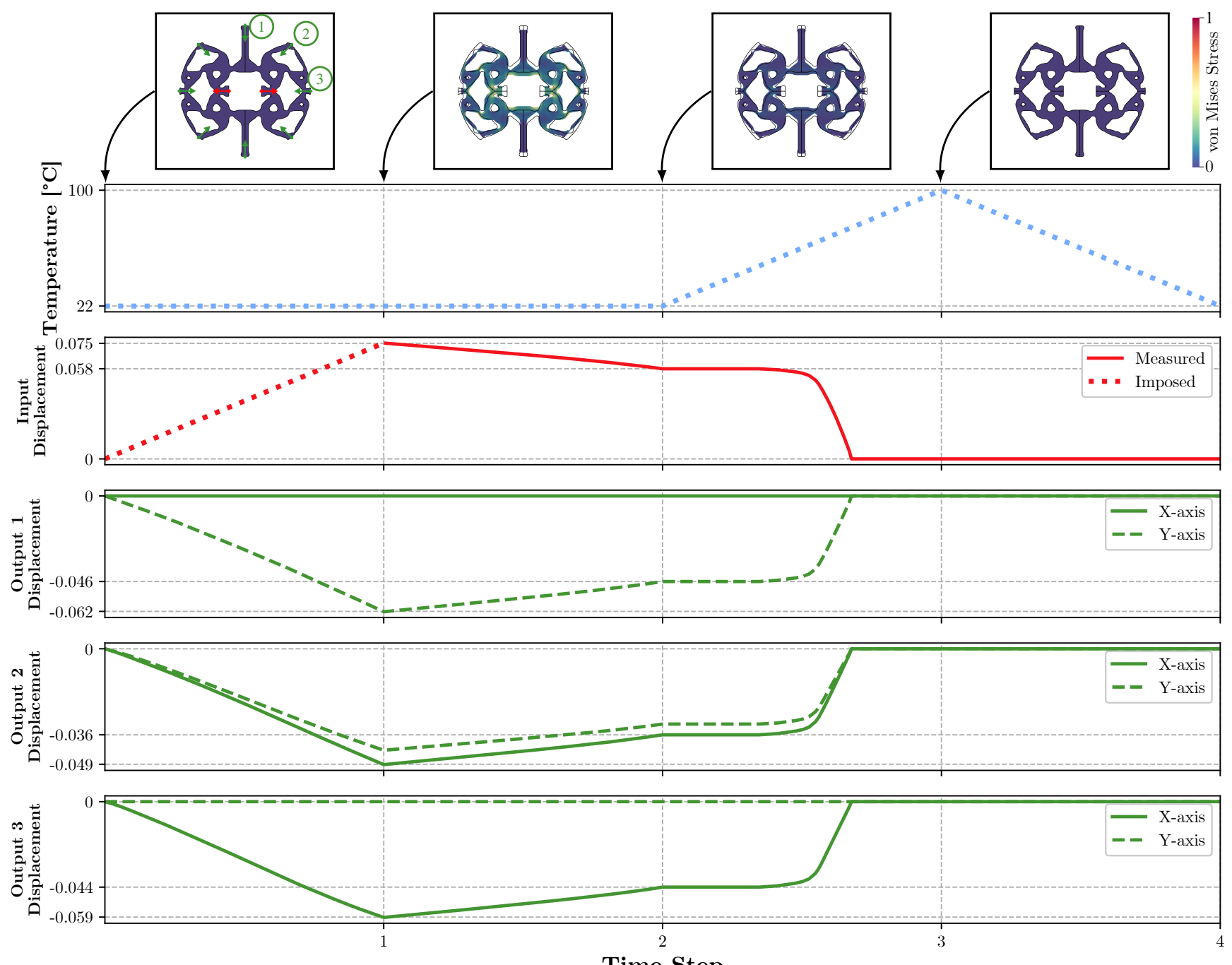

Time Step

Fig. 8: Results of the shape memory effect simulation of 8-point mandrel. Here, the displacements are calculated relatively to the design domain radius: $L / 2$. The displacements observed between time steps 1 and 2 show a strain retention of $\alpha_{\varepsilon}=78 \%$ confirming the presence of the shape memory effect. The outputs are numbered based on Fig. 9.

results of the SME simulation can be found in Fig. 6. The last example consists of a structure in which the bias-spring evokes a mandrel-like structure to contract radially towards the center at 8 points positions in a circle (with diameter: $L$ ) around the spring as seen in Fig. 9. The results of the simulation can be seen in Fig. 8 .

\section{DISCUSSION}

As shown in Fig. 5, even if the desired output displacement has an angle of $45^{\circ}$, the measured $x$ and $y$ output displacements have different magnitudes resulting in an angle of $40^{\circ}$, which corresponds to an error of $11 \%$. This is mainly due to the assumptions of small deformations and of linear elastic material that are done during the optimization process. To avoid this issue, one can strategically place the desired output displacements along symmetry axes as done in Fig. 6. This constrains the displacement along one axis and thus avoids any disturbances along the other direction. If not possible due to the application's requirements, one could
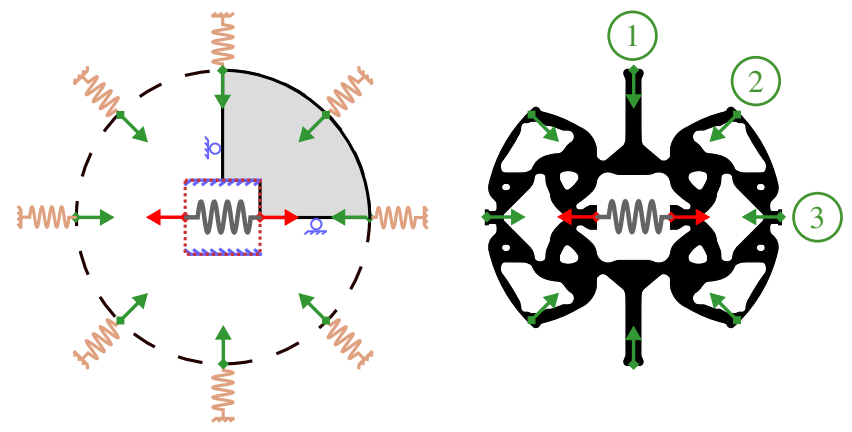

Fig. 9: 8-point Mandrel: Design problem (left), Interpolated final topology (right). Input forces are shown in red while the output forces are shown green.

implement the hyper-elastic properties of the SMA for the FEA performed inside the topology optimization. This would lead to non-linear analysis resulting in a significant increase in the computational time (especially important when shifting 
towards 3-dimensional cases) but would lead to more accurate designs. Some differences between the optimized behavior and the actual one may also come from the interpolation step when exporting the topology from its pixel-like definition to its physical one with smooth boundaries that is used in ANSYS. This error is minimized as the resolution of the discretization of the domain inside the topology optimization framework is increased.

This work does not dwell into the dimension of the bias-spring associated with each design. The work done to dimension the springs in traditional SMA actuators [18] can still be used to dimension them for these new designs. Future work can take into account the FEM simulations to obtain the required forces required for the detwinning process to optimize the geometry of the bias-spring. The proposed biased-spring compliant mechanisms made of SMA are designed assuming compression springs but all of the presented content stays valid for tension ones. A prototype of the proposed designs can be fabricated by water-jet cutting a thick sheet of SMA. This can be accomplished in a future work as a proof of concept for the design methodology. Finally, compliant mechanisms composed of rigid parts and only flexure hinges made of SMA could be developed. This would lead to a trade-off between better overall use of the material (as every SMA parts would be under strain) and increased complexity of the mechanism.

\section{CONCLUSION}

This work shows three promising compliant SMA biasspring actuators that can provide multi-output displacements using only one bias-spring. By eliminating the transducers that are normally adapted into traditional SMA bias-spring actuators, the resulting smart actuator is more compact and easier to assemble. The hope of this work is to replace traditional actuators that would require multiple complex pieces and assembly to provide the same kind of multi-output displacements [19].

By simulating the designs using FEM, the Shape Memory Effect and the mechanical behaviour of the structures were validated. Indeed, the work shows that the designs do in fact behave reversibly when actuated using heat.

\section{REFERENCES}

[1] J. Mohd Jani, M. Leary, A. Subic, and M. A. Gibson, "A review of shape memory alloy research, applications and opportunities," Materials \& Design (1980-2015), vol. 56, no. Supplement C, pp. 1078-1113, Apr. 2014. [Online]. Available: http://www.sciencedirect.com/science/article/pii/S0261306913011345

[4] Z. Zhakypov, F. Heremans, A. Billard, and J. Paik, "An OrigamiInspired Reconfigurable Suction Gripper for Picking Objects With Variable Shape and Size," IEEE Robotics and Automation Letters, vol. 3, no. 4, pp. 2894-2901, Oct. 2018. [Online]. Available: https://ieeexplore.iee.org/document/8385192/
[2] J.-H. Lee, Y. S. Chung, and H. Rodrigue, "Long Shape Memory Alloy Tendon-based Soft Robotic Actuators and Implementation as a Soft Gripper," Scientific Reports, vol. 9, no. 1, pp. 1-12, Aug. 2019. [Online]. Available: https://www.nature.com/articles/s41598019-47794-1

[3] R. Britz, P. Motzki, and S. Seelecke, "Scalable Bi-Directional SMA-Based Rotational Actuator," Actuators, vol. 8, no. 3, p. 60, Aug. 2019. [Online]. Available: https://www.mdpi.com/2076-0825/8/3/60

[5] R. Vitushinsky, S. Schmitz, and A. Ludwig, "Bistable Thin-Film Shape Memory Actuators for Applications in Tactile Displays," Journal of Microelectromechanical Systems, vol. 18, no. 1, pp. 186-194, Feb. 2009. [Online]. Available: http://ieeexplore.ieee.org/document/4729628/

[6] L. L. Howell, Handbook of Compliant Mechanisms. John Wiley \& Sons, Ltd, 2013.

[7] S. Henein, S. Bottinelli, and R. Clavel, "Parallel spring stages with flexures of micrometric cross sections," in Microrobotics and Microsystem Fabrication, A. Sulzmann, Ed., vol. 3202, International Society for Optics and Photonics. SPIE, 1998, pp. $209-220$. [Online]. Available: https://doi.org/10.1117/12.298039

[8] J.-P. Bacher, S. Bottinelli, J.-M. Breguet, and R. Clavel, "Delta3: design and control of a flexure hinge mechanism," in Microrobotics and Microassembly III, B. J. Nelson and J.-M. Breguet, Eds., vol. 4568, International Society for Optics and Photonics. SPIE, 2001, pp. 135 - 142. [Online]. Available: https://doi.org/10.1117/12.444120

[9] M. P. Bendsøe and O. Sigmund, Topology optimization: theory, methods, and applications, ser. Engineering online library. Berlin Heidelberg: Springer, 2011, oCLC: 711862335.

[10] B. Zhu, X. Zhang, H. Zhang, J. Liang, H. Zang, H. Li, and R. Wang, "Design of compliant mechanisms using continuum topology optimization: A review," Mechanism and Machine Theory, vol. 143, p. 103622 Jan. 2020.

[11] A. Thabuis, S. Thomas, T. Martinez, Y. Perriard, "Shape memory effect of benchmark compliant mechanisms designed with topology optimization," in Proceedings 2020 IEEE/ASME International Conference on Advanced Intelligent Mechatronics (AIM 2020), July 2020.

[12] Y. Bellouard, "Shape memory alloys for microsystems: A review from a material research perspective," Materials Science and Engineering: A, vol. 481-482, pp. 582-589, May 2008. [Online]. Available: http://www.sciencedirect.com/science/article/pii/S0921509307011318

[13] Z. W. Zhong and C. K. Yeong, "Development of a gripper using SMA wire," Sensors and Actuators A: Physical, vol. 126, no. 2, pp. 375-381, Feb. 2006. [Online]. Available: http://www.sciencedirect.com/science/article/pii/S0924424705005935

[14] P. Singh and G. K. Ananthasuresh, "A Compact and Compliant External Pipe-Crawling Robot," IEEE Transactions on Robotics, vol. 29, no. 1, pp. 251-260, Feb. 2013, conference Name: IEEE Transactions on Robotics.

[15] C. Alonso, R. Ansola, and O. M. Querin, "Topology synthesis of MultiInput-Multi-Output compliant mechanisms," Advances in Engineering Software, vol. 76, pp. 125-132, Oct. 2014.

[16] B. Zhu, Q. Chen, H. Li, H. Zhang, and X. Zhang, "Design of Planar Large-Deflection Compliant Mechanisms With Decoupled MultiInput-Output Using Topology Optimization," J. Mechanisms Robotics, vol. 11, no. 3, p. 031015, Apr. 2019.

[17] K. Svanberg, "The method of moving asymptotes - a new method for structural optimization," International Journal for Numerical Methods in Engineering, vol. 24, no. 2, pp. 359-373, Feb. 1987. [Online]. Available: https://onlinelibrary.wiley.com/doi/abs/10.1002/nme.1620240207

[18] S. Thomas, M. Almanza, and Y. Perriard, "Design Analysis of a Shape Memory Alloy Bias-Spring Linear Actuator," in 2019 12th International Symposium on Linear Drives for Industry Applications (LDIA), Jul. 2019, pp. 1-5, iSSN: null.

[19] W. Chen, S. Zhao, and S. L. Chow, "Grippers and End Effectors," in Handbook of Manufacturing Engineering and Technology, A. Nee, Ed. London: Springer London, 2014, pp. 1-32. 